\title{
VORTICES ON THE HIGGS BRANCH OF THE SEIBERG-WITTEN THEORY
}

\author{
Alexei Yung \\ Petersburg Nuclear Physics Institute \\ Gatchina, St.Petersburg 188350, Russia \\ E-mail: yung@thd.pnpi.spb.ru
}

June 1999

\begin{abstract}
We study the mechanism of confinement via formation of AbrikosovNielsen-Olesen vortices on the Higgs branch of $N=2$ supersymmetric $\mathrm{SU}(2)$ gauge theory with massive fundamental matter. Higgs branch represents a limiting case of superconductor of type I with vanishing Higgs mass. We show that in this limit vortices still exist although they become logarithmically "thick". Because of this the confining potential is not linear any longer. It behaves as $L / \log L$ with a distance $L$ between confining heavy charges (monopoles). This new confining regime can occur only in supersymmetric theories. We also address the problem of quantum stability of vortices. To this end we develop string representation for a vortex and use it to argue that vortices remain stable.
\end{abstract}

PNPI-TH-2319 


\section{Introduction}

Supersymmetric gauge theories can be viewed as a "theoretical laboratory" to develop insights in the dynamics of strongly coupled gauge theories like QCD. Revolutionary ideas of electromagnetic duality [1], 2] made it possible to study traditionally untractable strongly coupled theories using weak coupling description in terms of dual variables. Particularly spectacular results were obtained by Seiberg and Witten in $N=2$ supersymmetry where the low energy effective Lagrangians were found exactly [2, 3].

One of the most important physical outcome of the Seiberg-Witten theory is the demonstration of $\mathrm{U}(1)$ confinement via monopole condensation [2] According to old Mandelstam and t'Hooft ideas [4 when monopoles condense the electric flux is confined in the (dual) Abrikosov-Nielsen-Olesen (ANO) vortex tubes [5] connecting heavy trial charge-anticharge pair. Because this vortex has a constant energy per length (string tension $\tau$ ) the potential between charge and anticharge increases linearly with their separation. Thus, if monopoles (charges) condense then charges (monopoles) confine. The effective description of this phenomenon is given by the Abelian Higgs model.

The ratio of the Higgs mass $m_{H}$ to the photon mass $m_{\gamma}$ in the Abelian Higgs model is an important parameter characterizing the type of the super-

conductor. For $m_{H}>m_{\gamma}$ we have type II superconductor while for $m_{H}<m_{H}$ we have type I.

In the Seiberg-Witten theory this confinement scenario is realized in two possible ways. First, in pure gauge theory near monopole (dyon) singularity upon breaking $N=2$ supersymmetry to $N=1$ one by adding the mass term to the adjoint matter [2]. Second, in $N=2$ theory with fundamental matter hypermultiplets on Higgs branches [3].

Consider the $N=2, \mathrm{SU}(2)$ gauge theory [2]. The gauge group is broken down to $\mathrm{U}(1)$ by the expectation value $\left\langle\varphi^{a}\right\rangle=\delta^{a 3} a$ of the adjoint scalar field $\varphi^{a}, a=1,2,3$. The complex parameter $a$ parametrize the modular space (Coulomb branch) of the theory. Near the monopole point on the Coulomb branch (the point, where monopole becomes massless) the effective low energy theory is the dual $N=2$ QED. This means that the theory has light monopoles coupled to dual photon in the same way as ordinary charges are coupled to the ordinary photon. 
Upon breaking $N=2$ supersymmetry by the mass term for the adjoint matter monopoles condense implying the $\mathrm{U}(1)$ confinement for heavy trial charges. The effective low energy theory is given by the Abelian Higgs model with $m_{H}=m_{\gamma}$ [6] (here $m_{H}$ is the mass of the monopole and $m_{\gamma}$ is the mass of the dual photon). This condition means that the ANO vortex saturate the Bogomolny bound [7] (see [8] for the relation between supersymmetry and the BPS bound) and the string tension $\tau$ is given by

$$
\tau=2 \pi v^{2}
$$

at least for small masses of the adjoint matter. Here $v$ is the VEV of the Higgs field.

The second option is the confinement on Higgs branches in the SeibergWitten theory. If two (or more) flavours of matter of the $N=2 \mathrm{SU}(2)$ gauge theory with $N_{f}$ fundamental hypermultiplets have the same mass then the Higgs branch is developed with $\langle Q\rangle \neq 0$, where $Q$ is the matter hypermultiplet [3]. Positions of roots of Higgs branches on the Coulomb branch depend on the mass parameter $m$. The low energy theory near a given singularity (root) is $N=2$ Abelian Higgs model, in which the role of the Higgs hypermultiplet is played by the state becoming massless in this singularity.

Once charges (monopoles) condense on the Higgs branch we expect monopoles (charges) to confine [3, 9]. However, this scenario is much less understood, than the first one discussed above. The purpose of the present paper is to study the mechanism of the confinement on Higgs branches via formation of ANO vortices. We consider the case of two hypermultiplets $N_{f}=2$ with the common mass $m$ for simplicity.

From the point of view of the low energy Abelian Higgs model the Higgs branch is a limiting case of the superconductor of type I with $m_{H}=0$. The usual arena for studies of ANO vortices is the opposite, so called London limit of type II superconductivity with $m_{H} \gg m_{\gamma}$. In the London limit the string tension is given by

$$
\tau_{I I}=2 \pi v^{2} \ln \frac{m_{H}}{m_{\gamma}} .
$$

\footnotetext{
${ }^{1}$ It is argued in [9] that this string is not in fact the BPS-saturated state (it does not belong to the "short" multiplet) due to higher corrections in the mass of the adjoint matter.
} 
This result was obtained by Abrikosov in 1957 and then rederived by Nielsen and Olesen in the framework of the relativistic field theory [5].

In this paper we study vortices in the limit $m_{H}=0$. Classical vortex solutions still exist for the type I superconductor [10 and we show that they survive the limit $m_{H}=0$. However, their transverse size becomes logarithmically large. As a result the string tension becomes suppressed as

$$
\tau=\frac{2 \pi v^{2}}{\ln m_{\gamma} L}
$$

where $L$ is the length of the string (the distance between heavy monopole and antimonopole). In fact, $\tau$ in (1.3) goes to zero for infinite strings in accordance with [11].

Our result (1.3) means quite unusual behavior from the point of view of confinement. Eq.(1.3) means that the confining potential between well separated heavy sources (monopole and antimonopole in the case at hand) is not linear in $L$ any longer. It behaves as

$$
V(L)=2 \pi v^{2} \frac{L}{\ln m_{\gamma} L}
$$

for large $L$.

This peculiar behavior is specific for supersymmetric gauge theories with Higgs branches (we don't have Higgs branches with $m_{H}=0$ without supersymmetry). The potential (1.4) is the order parameter which distinguish this new confinement phase from the ordinary confinement phase with $V(L) \sim L$ or from other phases. The potential (1.4) gives rise to the following behavior of the Wilson loop produced by the infinitely heavy monopole going along the contour $C$. For the $W_{C}^{D}=P \exp i \int_{C} d x_{\mu} A_{\mu}^{D} \quad\left(A_{\mu}^{D}\right.$ is the dual potential) the obvious generalization of (1.4) gives

$$
W_{C}^{D}=\exp \left\{-\frac{2 \pi^{2} v^{2} S}{\ln \left(m_{\gamma} \cdot 2 S / P\right)}\right\}
$$

for large $S$ and $P$, where $S$ is the area spanned by the loop $C$ and $P$ is its perimeter. For rectangular loops $S=T L$ and $P=2(L+T)$, where $T$ is the time interval. We see that (1.5) is different from the usual area law for the Wilson loop. 
The paper is organized as follows. In Sect.2 we review the Higgs branches in the $N_{f}=2$ Seiberg-Witten theory and derive the low energy action on the Higgs branch. In Sect.3 we study classical vortex solution and derive the result (1.3). Then in Sect.4 we address the problem of quantum stability of vortices. We develop string representation for the vortex and use it to argue that vortices survive in the quantum theory. Sect. 5 contains our conclusions.

\section{Review of Higgs branches in $\mathrm{SU}(2)$ theory}

Let us introduce $N_{f}=2$ fundamental matter hypermultiplets in the $N=2$ $\mathrm{SU}(2)$ gauge theory. In terms of $N=1$ superfields matter dependent part of the microscopic action looks like

$$
\begin{gathered}
S_{\text {matter }}=\int d^{4} x d^{2} \theta d^{2} \bar{\theta}\left[\bar{Q}_{A} e^{V} Q^{A}+\overline{\widetilde{Q}}^{A} e^{V} \widetilde{Q}_{A}\right]+ \\
+\quad i \int d^{4} x d^{2} \theta\left[\sqrt{2} \widetilde{Q}_{A} \frac{\tau^{a}}{2} Q^{A} \Phi^{a}+m \widetilde{Q}_{A} Q^{A}\right]+\text { c.c. }
\end{gathered}
$$

Here $Q^{k A}, \widetilde{Q}_{A k}$ are matter chiral fields, $k=1,2$ and $A=1, \ldots, N_{F}$, while $V$ is the vector superfield. $\Phi^{a}$ is the adjoint chiral superfield (its scalar component $\varphi^{a}$ develops VEV a). Thus we have 16 real matter degrees of freedom for $N_{f}=2$.

Consider first the limit of large $m, m \gg \Lambda$. Then three singularities on the Coulomb branch are easy to understand. Two of them correspond to monopole and dyon singularities of the pure gauge theory. Their positions on the Coulomb branch are given by [3]

$$
u_{m, d}= \pm 2 m \Lambda-\frac{1}{2} \Lambda^{2}
$$

where $u=\frac{1}{2}\left\langle\varphi^{a^{2}}\right\rangle \simeq a^{2} / 2$. I In the large $m$ limit $u_{m, d}$ are approximately given by their values in the pure gauge theory $u_{m, d} \simeq \pm 2 m \Lambda= \pm 2 \Lambda_{0}^{2}$, where $\Lambda_{0}$ is the scale of $N_{f}=0$ theory.

\footnotetext{
${ }^{2}$ We use the Pauli-Villars regularization scheme for the normalization of $\Lambda$, see [12].
} 
The third singularity corresponds to the point where charge becomes massless. Let us decompose matter fields as

$$
Q^{k A}=\left(\begin{array}{l}
1 \\
0
\end{array}\right)^{k} Q_{+}^{A}+\left(\begin{array}{l}
0 \\
1
\end{array}\right)^{k} Q_{-}^{A}
$$

From the superpotential in (2.1) we see that the $Q_{+}$becomes massless at

$$
a=-\sqrt{2} m
$$

The singular point $a=+\sqrt{2} m$ is gauge equivalent to the one in (2.4). In terms of variable $u(2.4)$ reads

$$
u_{c}=m^{2}+\frac{1}{2} \Lambda^{2}
$$

Strictly speaking, we have $2+N_{f}=4$ singularities on the Coulomb branch. However two of them coincides for the case of two flavors of matter with the same mass.

The effective theory on the Coulomb branch near charge singularity (2.4) is given by $N=2$ QED with light matter fields $Q_{+}^{A}, \widetilde{Q}_{+A}$ (8 real degrees of freedom) as well as the photon multiplet.

The charge singularity $(2.4),(2.5)$ is the root of the Higgs branch [3]. To find this branch let us write down $D$-term and $F$-term conditions which follow from (2.1). D-term conditions are

$$
Q^{k A} \bar{Q}_{A \ell}+\overline{\widetilde{Q}}^{k A} \widetilde{Q}_{A \ell}=0
$$

while $F$-term conditions give (2.4) as well as

$$
Q^{k A} \widetilde{Q}_{A \ell}=0
$$

Eqs. (2.6),(2.7) have nontrivial solutions for $N_{f} \geq 2$. These solutions determines VEV's for scalar components $q^{k A}, \widetilde{q}_{A k}$ of fields $Q^{k A}, \widetilde{Q}_{A k}$. Dropping heavy components $q_{-}$according to decomposition (2.3) and introducing the $\mathrm{SU}_{R}(2)$ doublet $q^{f A}$ as

$$
\begin{array}{ll}
q^{1 A}=q_{+}^{A}, & q^{2 A}=\overline{\widetilde{q}}_{+}^{A} \\
\bar{q}_{A 1}=\bar{q}_{A}^{+}, & \bar{q}_{A 2}=-\widetilde{q}_{A}^{+}
\end{array}
$$


we can rewrite three real conditions in $(2.6),(2.7)$ as

$$
\bar{q}_{A p}\left(\tau^{a}\right)_{f}^{p} q^{f A}=0, \quad a=1,2,3 .
$$

Eq.(2.9) together with the condition (2.4) determines the Higgs branch (manifold with $\langle q\rangle \neq 0$ ) which touches the Coulomb branch at the point (2.4).

The low energy theory for boson fields near the root of the Higgs branch looks like

$$
\left.S_{\text {boson }}^{\text {root }}=\int d^{4} x\left\{\frac{1}{4 g^{2}} F_{\mu \nu}^{2}+\bar{\nabla}_{\mu} \bar{q}_{A f} \nabla_{\mu} q^{f A}+\frac{g^{2}}{8}\left[\operatorname{Tr} \bar{q} \tau^{a} q\right)\right]^{2}\right\},
$$

where trace is calculated over flavor and $\mathrm{SU}_{R}(2)$ indices. Here $\nabla_{\mu}=\partial_{\mu}-$ $i n_{e} A_{\mu}, \bar{\nabla}_{\mu}=\partial_{\mu}+i n_{e} A_{\mu}$, the electric charge $n_{e}=1 / 2$ for fundamental matter fields.

This is an Abelian Higgs model with last interaction term coming from the elimination of $D$ and $F$ terms. The QED coupling constant $g^{2}$ is small near the root of Higgs branch (we specify its value later on). We include 8 real matter degrees of freedom $q^{f A}$ in the theory $(2.10)$ according to the identification (2.8). The rest of matter fields $q_{-}^{A}, \widetilde{q}_{\bar{A}}^{-}$(another 8 real degrees of freedom) acquire large mass $2 m$ and can be dropped out. The effective theory (2.10) is correct on the Coulomb branch near the root of the Higgs branch (2.4) or on the Higgs branch not far away from the origin $\langle q\rangle=0$.

It is clear that the last term in (2.10) is zero on the fields $q$ which satisfy constraint (2.9). This means that moduli fields which develop VEV's on the Higgs branch are massless, as it should be. The other fields acquire mass of order $\langle\bar{q} q\rangle^{1 / 2}$. It turns out that there are four real moduli fields $q$ (out of 8 ) which satisfy the constraint (2.9) [3]. They correspond to lowest components of the one hypermultiplet.

We can parametrize them as

$$
q^{f \dot{A}}(x)=\frac{1}{\sqrt{2}} \sigma_{\alpha}^{f \dot{A}} \phi_{\alpha}(x) e^{i \alpha(x)}
$$

Here $\phi_{\alpha}(x), \alpha=1 \ldots 4$ are four real moduli fields. It is clear that fields $(2.11)$ solve (2.9). The common phase $\alpha(x)$ in (2.11) is the $\mathrm{U}(1)$ gauge phase. Once $\left\langle\phi_{\alpha}\right\rangle=v_{\alpha} \neq 0$ on the Higgs branch the $\mathrm{U}(1)$ group is broken and $\alpha(x)$ is 
eaten by the Higgs mechanism. Say, in the unitary gauge $\alpha(x)=0$. In the next section we consider vortex solution for the model (2.10). Then $\alpha(x)$ is determined by the behavior of the gauge field at the infinity. Substituting (2.11) into (2.10) we get the bosonic part of the effective theory on the Higgs branch near the origin

$$
S_{\text {boson }}^{\text {Higgs }}=\int d^{4} x\left\{\frac{1}{4 g^{2}} F_{\mu \nu}^{2}+\bar{\nabla}_{\mu} \bar{q}_{\alpha} \nabla_{\mu} q_{\alpha}\right\},
$$

where

$$
q_{\alpha}(x)=\phi_{\alpha}(x) e^{i \alpha(x)}
$$

Once $v_{\alpha} \neq 0$ we expect monopoles (they are heavy at $m \gg \Lambda$ ) to confine via formation of vortices which carry the magnetic flux. In the rest of the paper we study in detail vortex solutions in the model (2.12). As we mentioned in the Introduction this model corresponds to the type I superconductivity with the vanishing Higgs mass, $m_{H}=0$. The photon mass in the model $(2.12)$ is

$$
m_{\gamma}^{2}=\frac{1}{2} g^{2} v_{\alpha}^{2}
$$

Note, that we do not introduce Fayet-Illiopoulos term in the SeibergWitten theory. In the presence of such term there are BPS-saturated vortices. They correspond to special points on the modular space where VEV's of massless moduli fields $\left\langle q_{\alpha}\right\rangle=0$, whereas other massive matter fields from (2.10) develop VEV's equal to Fayet-Illiopoulos parameters with windings at spatial infinity [15, 9]. Instead, vortices which arise in theories with flat Higgs potential are not BPS-saturated. They are discussed from the brane point of view in [16] and from the field theory point of view in [11].

If we increase $v_{\alpha}^{2}$ taking $v_{\alpha}^{2} \gtrsim \Lambda^{2}$ we can integrate out massive photon. Then the effective theory is a $\sigma$-model for massless fields $q_{\alpha}$ which belong to 4-dimensional Hyper-Kahler manifold, $R^{4} / Z_{2}$. The metric of this $\sigma$-model is flat [3, 13], there are, however, higher derivative corrections induced by instantons [14. In this paper we consider region of Higgs branch with $v_{\alpha}^{2} \ll$ $\Lambda$. This determines the scale of the effective Abelian Higgs model (2.12). $W$-bosons and other particles which reflect the non-Abelian structure of the underlying microscopic theory are heavy with masses $\gtrsim \Lambda$ and can be ignored. 
To conclude this section let us briefly review what happens if we reduce the mass parameter $m$. At $m= \pm \Lambda$ the charge singularity (root of the Higgs branch) collides with the monopole (dyon) singularity, see Eqs.(2.2),(2.5). These are Argyres-Douglas points [17, 18]. At these points mutually non-local degrees of freedom (say, charges and monopoles) becomes massless simultaneously. These points are very interesting from the point of view of the monopole confinement on the Higgs branch we study in this paper. Monopoles become dynamical as we approach Argyres-Douglas point, $m \rightarrow \Lambda$. We comment on the physics at this point at the end of the paper.

After the collision quantum numbers of particles at singularities change because of monodromies [3]. If we denote quantum numbers as $\left(n_{m}, n_{e}\right)_{B}$, where $n_{m}$ and $n_{e}$ are magnetic and electric charges of the state, while $B$ is its baryon number then at $m>\Lambda$ we have charge, monopole and dyon singularities with quantum numbers

$$
(0,1 / 2)_{1}^{* 2}, \quad\left(\begin{array}{lll}
1 & 0
\end{array}\right)_{0}, \quad(1,1)_{0} .
$$

The superscript for the charge means that we have two flavors of charges. After charge singularity collides with monopole one (at $m<\Lambda$ ) the quantum numbers of particles at singularities become [19]

$$
(1,0)_{0}^{* 2}, \quad(1,1 / 2)_{1}, \quad(1,1 / 2)_{-1} .
$$

Now monopole $(1,0)_{0}$ condense on the Higgs branch which emerges from the point $(2.5)$, while dyons $(1,1 / 2)_{1}$ and $(1,1 / 2)_{-1}$ confine because they carry electric charge. 3 At zero mass, $m=0$ two dyon singularities in (2.16) coincide (see (2.2)) and the second Higgs branch appears at the point $u=-1 / 2 \Lambda^{2}$. This restores the global symmetry from $\mathrm{SU}\left(N_{f}=2\right)$ in the massive theory to $\operatorname{SO}\left(2 N_{f}=4\right)$ at $m=0 \quad[3$.

\section{Vortex solution}

In this section we construct the classical vortex solution for the model (2.12). Without loss of generality we can rotate VEV's $v_{\alpha}$ in the $R^{4}$ space to the

\footnotetext{
${ }^{3}$ Throughout the rest of the paper to avoid confusion we refer to particles which develop VEV's on the Higgs branch as charges and to states which confined as monopoles (dyons). This terminology corresponds to masses of matter above Argyres-Douglas point $m>\Lambda$. At $m<\Lambda$ one should change the terminology according to Eqs. (2.15),(2.16).
} 
configuration $v_{\alpha}=(v, 0,0,0)$. Now we look for vortex solutions with $q_{1} \equiv$ $q \neq 0$ and $q_{2}=q_{3}=q_{4}=0$. Thus for the purpose of finding the classical vortex solution we can drop fields $q_{2}, q_{3}, q_{4}$ in (2.12) f. Doing so we arrive at the standard Abelian Higgs model with one complex matter field $q \equiv q_{1}$

$$
S_{A H}=\int d^{4} x\left\{\frac{1}{4 g^{2}} F_{\mu \nu}^{2}+\bar{\nabla}_{\mu} \bar{q} \nabla_{\mu} q+\lambda\left(|q|^{2}-v^{2}\right)^{2}\right\} .
$$

Here we write down the double well potential term for the matter field $q$ with coupling constant $\lambda$. We cannot introduce this term in the Seiberg-Witten theory without breaking down $N=2$ supersymmetry. We use this term to regularize the vortex solution and afterwards take the limit $\lambda \rightarrow 0$. In terms of $\lambda$ the mass of the Higgs field reads

$$
m_{H}^{2}=4 \lambda v^{2} .
$$

We consider the model (3.1) at

$$
m_{H} \ll m_{\gamma},
$$

(see (2.14)) taking the limit $m_{H} \rightarrow 0$ in the end. The limit (3.3) is the opposite to the London one.

\subsection{Two dimensional problem}

To begin with in this subsection we study infinitely long ANO vortex, with length $L \gg 1 / m_{H}$. In fact, this is not what we want. To take limit $m_{H} \rightarrow 0$ we have to study not that long vortices. We focus on the opposite limit $L \ll 1 / m_{H}$ in the next subsection.

For infinitely long vortex the problem becomes two dimensional. Assume that solutions for $q$ and $A_{\mu}$ depend only on $x_{i}, i=1,2$. Let us use the standard ansatz for fields $q$ [7]

$$
q(x)=\phi(r) e^{i \arg \left(x^{i}\right)},
$$

where $\phi(r)$ is real, and for $A_{\mu}$

$$
n_{e} A_{i}(x)=-\varepsilon_{i j} \frac{x_{j}}{x^{2}}[1-f(r)],
$$

\footnotetext{
${ }^{4}$ We cannot ignore these massless fields in the quantum theory, however.
} 
where $r^{2}=x_{i}^{2}$, while $A_{0}=A_{3}=0$. We also assume standard boundary conditions for $\phi(r)$

$$
\begin{aligned}
& \phi(\infty)=v, \\
& \phi(0)=0,
\end{aligned}
$$

and

$$
\begin{aligned}
& f(0)=1 \\
& f(\infty)=0
\end{aligned}
$$

for $f(r)$.

The boundary conditions (3.7) for gauge field ensures that the vortex carry one unit of the magnetic flux. To see this let us calculate the dual field strength

$$
F^{*}=\varepsilon_{i j} \partial_{i} A_{j}=-\frac{1}{n_{e} r} f^{\prime}(r) .
$$

Then using (3.7) one gets

$$
n_{e} \int d^{2} x F^{*}=2 \pi
$$

Substituting (3.4), (3.5) into the action (3.1) we get the energy of the vortex as a function of $\phi$ and $f$

$$
\varepsilon=2 \pi L \int d r\left\{\frac{1}{2 n_{e} g^{2}} \frac{f^{\prime 2}}{r}+r \phi^{\prime 2}+\frac{f^{2}}{r} \phi^{2}+\lambda\left(\phi^{2}-v^{2}\right)^{2} r\right\} .
$$

The equations of motion read for field $\phi$

$$
\phi^{\prime \prime}+\frac{1}{r} \phi^{\prime}-\frac{1}{r^{2}} f^{2} \phi-m_{H}^{2} \frac{\phi\left(\phi^{2}-v^{2}\right)}{2 v^{2}}=0
$$

and for $f$

$$
f^{\prime \prime}-\frac{1}{r} f^{\prime}-\frac{m_{\gamma}^{2}}{v^{2}} \phi^{2} f=0
$$

Now to solve these equations in the limit $m_{\gamma} \gg m_{H}$ we adopt the following model for the vortex. We assume that the electromagnetic field of the vortex is confined inside the core of radius $R_{g}$ (we find $R_{g}$ later), such that $F^{*}=0$ 
at $r \geq R_{g}$. Instead scalar field $\phi$ is almost zero inside the core and grows towards its $\operatorname{VEV} \phi=v$ at the infinity outside the core.

Thus at zero order approximation we assume that $\phi=0$ at $r \leq R_{g}$. We improve this approximation later on and show that corrections to $\phi$ inside the core do not affect the string energy in the logarithmic approximation.

If $\phi=0$ inside the core we have from Eq.(3.12) for gauge field $f$

$$
f^{(0)}=\left[\begin{array}{cl}
1-\frac{r^{2}}{R_{g}^{2}}, & r \leq R_{g} \\
0, & r \geq R_{g} .
\end{array}\right.
$$

The superscript (0) means the zero order approximation. To this order the magnetic field is constant inside the core and zero outside, see (3.8).

Now consider Eq.(3.11) for $\phi(r)$ outside the core. Taking into account (3.13) and neglecting the nonlinearity in (3.11) at large $r$ we find that (3.11) reduces to the equation of motion of free field with mass $m_{H}$. We have at $r \gg R_{g}$

$$
(\phi-v) \sim-v K_{0}\left(m_{H} r\right) .
$$

Here $K_{0}$ is a Bessel function. It has exponential fall-off at infinity $K_{0}\left(m_{H} r\right) \sim$ $e^{-m_{H} r}$ and logarithmic behavior at small $m_{H} r$ :

$$
K_{0}\left(m_{H} r\right) \simeq \ln \frac{2}{m_{H} r} .
$$

We assume that $R_{g} \ll 1 / m_{H}$ (we confirm this later) so $K_{0}\left(m_{H} r\right)$ becomes very large at $r \sim R_{g}$. To match the solution (3.14) with the value $\phi=0$ inside the core we have to normalize (3.14) properly. Doing so we finally get for the field $\phi$

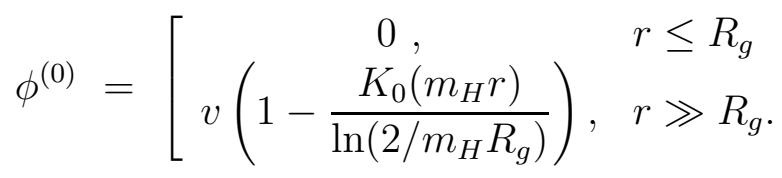

\footnotetext{
${ }^{5}$ As soon as we are going to take the limit $m_{H} \rightarrow 0$ the last term in (3.11) plays really the role of a mass term which regularize the behavior of $\phi$ at $r \rightarrow \infty$ and the nonlinearity associated with this term is not important.
} 
Substituting (3.13) and (3.16) into (3.10) we get the energy of vortex as a function of $R_{g}$

$$
\varepsilon=2 \pi L\left\{\frac{8}{g^{2}} \frac{1}{R_{g}^{2}}+\frac{v^{2}}{\ln \left(2 / m_{H} R_{g}\right)}\left[1+0\left(\frac{1}{\ln \left(1 / m_{H} R_{g}\right)}\right)\right]\right\} .
$$

The first term here comes from the electromagnetic energy inside the core (see the first term in (3.10)). The second term comes from the scalar field "surface energy" (the second term in(3.10)). All other terms are small in powers of $1 / \ln \left(m_{H} R_{g}\right)$. Note, that the second term in (3.17) comes from the logarithmic integration in the region $R_{g} \ll r \ll 1 / m_{H}$. This logarithm in the numerator cancels one of two logarithms in the denominator (coming from the normalization in (3.16)) giving the result (3.17).

Minimizing (3.17) with respect to $R_{g}$ we find

$$
R_{g}^{2} \simeq \frac{8}{m_{\gamma}^{2}} \ln ^{2} \frac{m_{\gamma}}{m_{H}}
$$

Substituting this into (3.17) we get finally the vortex energy

$$
\varepsilon_{0}=\frac{2 \pi v^{2}}{\ln \left(m_{\gamma} / m_{H}\right)} L .
$$

We stress once again that the main contribution to the vortex energy (3.19) comes from the logarithmic "tail" (3.16) of the scalar field outside the core.

So far we have considered vortices with winding number $n=1$. Now it is clear that to the leading order the vortex energy does not depend on $n$ for $m_{H} \ll m_{\gamma}$ and is given by Eq.(3.19).

We can calculate corrections to our zero order approximation vortex (3.13),(3.16) in the intermediate region $r \sim R_{g}$. For example, taking into account (3.13) in Eq.(3.11) we get the improved behavior for $\phi(r)$ inside the core $r \lesssim R_{g}$

$$
\phi(r)=\frac{v}{\ln \left(2 / m_{H} R_{g}\right)+1} \frac{r}{R_{g}}+\cdots,
$$

where dots stand for higher powers of $r / R_{g}$.

In a similar way we can get the next-to-leading correction to $f(r)$ at small $r$

$$
f(r)=1-\frac{r^{2}}{R_{g}^{2}}+\frac{1}{8} \frac{r^{4}}{R_{g}^{4}}+\cdots
$$


It is easy to see, however, that these corrections do not affect the main contribution to the energy of the vortex (3.19) in the logarithmic approximation.

Now let us discuss the result (3.18),(3.19). If we formally take the limit $m_{H} \rightarrow 0$ then the vortex becomes infinitely "thick" $\left(R_{g} \rightarrow \infty\right)$ and its string tension goes to zero. This result is in the accordance with results of Ref.[11], where it was noted that there are no vortices for the theories with flat Higgs potential. The reason is that field $\phi$ has logarithmic behavior outside the core and cannot approach its boundary condition $\phi=v$ at infinity. This can be seen from (3.16). If we put the IR-regularization $m_{H}$ to zero in (3.16) then $K_{0}\left(m_{H} r\right)$ is given by (3.15) and $\phi$ goes to infinity instead of $v$ at $r \rightarrow \infty$.

It is clear therefore, that the infinitely long vortex does not exist at $m_{H}=$ 0 P In the next subsection we consider vortices of the finite length $L$ and show that $L$ plays the role of IR-regularization in the limit $m_{H}=0$.

\subsection{Three dimensional problem}

Now, we are going to consider long but not infinitely long vortices $1 / m_{\gamma} \ll$ $L \ll 1 / m_{H}$. This will allow us to take the limit $m_{H} \rightarrow 0$.

As we already mentioned before the main problem with the limit $m_{H} \rightarrow 0$ is that in this limit the field $\phi$ outside the core obeys free field equation (see (3.11)). Thus $\phi \sim \ln r$ at large $r$ and cannot approach its boundary value $v$ at $r \rightarrow \infty$.

However, it is clear that for the vortex of finite length $L$ at $|x| \gg L$ field $\phi$ behaves as $\phi-v \sim 1 /|x|$. Thus it can reach its boundary value at infinity.

Let us consider the vortex of length $L$ stretched in $x_{3}$ direction between static heavy monopole at the point $x_{3}=L / 2, x_{1}=x_{2}=0$ and antimonopole at $x_{3}=-L / 2, x_{1}=x_{2}=0$. More specifically we consider the same ansatz (3.4),(3.5) for fields $q$ and $A_{\mu}$ with winding phase $\alpha(x)=\arg \left(x_{1}, x_{2}\right)$ for $\left|x_{3}\right| \ll L$ and $\alpha=0$ for $\left|x_{3}\right| \gg L$. Functions $\phi$ and $f$ now depend on $r^{2}=x_{1}^{2}+x_{2}^{2}$ and $x_{3}$.

The electromagnetic field is confined in the narrow tube of radius $R_{g}$ (we determine $R_{g}$ later) which is stretched between monopole and antimonopole.

\footnotetext{
${ }^{6}$ See, however, the discussion of tensionless strings in the brane picture [20].
} 
We assume the same behavior for $f(r)$ as in (3.13) at $\left|x_{3}\right| \ll L$ and put $f=0$ at $\left|x_{3}\right| \gg L$. For field $\phi$ we have $\phi=0$ at $r<R_{g}$ (with possible corrections like in (3.20)), $\phi-v \sim \ln r / L$ at $R_{g} \ll r \ll L$ and $(\phi-v) \sim-1 /|x|$ at $|x| \gg L$.

Normalizing these solutions to fit them together in the intermediate regions $r \sim R_{g}$ and $|x| \sim L$ we get

$$
\phi^{(0)}=\left\{\begin{array}{l}
0, \quad r \leq R_{g}, \quad x_{3} \ll L \\
v-\frac{v}{\ln L / R_{g}} \ln \frac{L}{r}, \quad R_{g} \ll r \ll L, \quad x_{3} \ll L \\
v-c \frac{v}{\ln L / R_{g}} \frac{L}{|x|} e^{-m_{H}|x|}, \quad|x| \gg L
\end{array}\right.
$$

where constant $c \sim 1$.

Substituting (3.13),(3.22) into the action (3.1) we get the vortex energy as a function of $R_{g}$

$$
\varepsilon=2 \pi L\left\{\frac{8}{g^{2}} \frac{1}{R_{g}^{2}}+\frac{v^{2}}{\ln \left(L / R_{g}\right)}\left[1+0\left(\frac{1}{\ln \left(L / R_{g}\right)}\right)\right]\right\} .
$$

Here the first term comes from the electromagnetic energy in (3.1) and the second one from the kinetic energy of field $\phi$ ("surface energy") in the region of logarithmic integration $R_{g} \ll r \ll L$. Minimizing (3.23) with respect to $R_{g}$ we get

$$
R_{g}^{2}=\frac{8}{m_{\gamma}^{2}} \ln ^{2} m_{\gamma} L
$$

and the vortex energy

$$
\varepsilon_{0}=2 \pi v^{2} \frac{L}{\ln m_{\gamma} L},
$$

which gives our result (1.3) for the string tension $\tau=\varepsilon_{0} / L$.

Again it is easy to see that corrections to functions $f$ and $\phi$ in the intermediate regions $\gamma \sim R_{g}$ and $|x| \sim L$ do not change (3.25). Moreover, we can make our model for the vortex more complicated. Introduce another free parameter $R_{0}$ (besides $R_{g}$ ) and write down

$$
\phi=v-\frac{v}{\ln \left(R_{0} / R_{g}\right)} \ln \frac{R_{0}}{r},
$$


at $R_{g} \ll r \ll L$. This region gives the main contribution to the energy. Minimizing the energy with respect to $R_{0}$ we find $R_{0} \sim L$ and the vortex energy is still given by (3.25).

Our results in (3.24), (3.25) show that the vortex length $L$ really plays the role of the IR-cutoff. Now we can safely put $m_{H}=0$. The radius of the vortex remains finite and its energy nonzero.

The result in (3.25) means that monopoles are in the confinement phase on the Higgs branch of the Seiberg-Witten theory. However, the confinement potential is not linear in $L$. It shows weaker behavior $L / \log L$ at large $L$. This new confinement phase is a specific one for supersymmetric theories with Higgs branches.

Of course, results of this section are purely classical. In the next section we address the problem of quantum stability of vortices on the Higgs branch.

\section{Quantum stability of vortices}

\subsection{General considerations}

In a given Higgs vacuum with $\langle\bar{q} q\rangle \neq 0$ the stability of vortices is ensured by the topology. Vortices correspond to minimum energy configurations with a given winding number $n$, which is an element of $\pi_{1}(U(1))=Z$.

However, one might suspect that the presence of vortices can destabilize the Higgs branch causing the phase transition to the unbroken phase with $\langle\bar{q} q\rangle=0$. The reason for this is that in the theory with flat Higgs potential there is no loss of volume energy associated with transition from the broken phase with $\langle\bar{q} q\rangle \neq 0$ to the unbroken phase with $\langle\bar{q} q\rangle=0$.

Let us compare two configurations. First is the monopole-antimonopole pair at large separation $L$ in the Higgs vacuum $\langle\bar{q} q\rangle=v^{2} \neq 0$. The magnetic flux of the monopole creates the vortex string connecting the monopole with the antimonopole. The energy of this configuration is given by (3.25). Sec-

\footnotetext{
${ }^{7}$ As we have shown in the last section the vortex energy does not depend on winding number $n$ in the limit $m_{H}=0$. Thus vortex with winding number $n$ has less energy than $n$ vortices with $n=1$. We discuss this issue in the subsection 4.3.
} 
ond, consider the same monopole-antimonopole pair in the unbroken vacuum with $\langle\bar{q} q\rangle=0$. The energy of this configuration associated with Coulomb interaction of monopole and antimonopole is

$$
\varepsilon \sim \frac{1}{L},
$$

with possible logarithmic corrections coming from the renormalization of the coupling constant.

Comparing (3.25) and (4.1) at large $L$ one can see that the monopoleantimonopole pair in the unbroken vacuum has less energy. Therefore, one may suspect that the presence of vortices induces the collapse of the Higgs branch with the phase transition to the Coulomb branch with $\langle\bar{q} q\rangle=0$.

Now it is clear that the problem is whether the barrier of this transition is finite or infinite. We argue in this section that the barrier is in fact infinite and the Higgs branch remains stable.

Another possible way to understand our conclusion about the stability of vortices we are going to make in this section is to note that the estimate (4.1) is hardly correct. The point is that once the "electric" coupling constant $g^{2}$ is small then the "magnetic" one is large. This means that there are uncontrollable corrections to the Coulomb interactions of the monopole and the antimonopole in (4.1).

Of course, we cannot prove that the vortex is stable in the quantum theory (still, we present some general arguments in subsection 4.3). However, in this section we study several natural possibilities for the vortex to develop instability and show that they do not lead to the collapse of the Higgs branch.

First, imagine that the radius of the vortex $R_{g}$ grows and at large $R_{g}$ the vortex becomes a bubble of the unbroken vacuum inside the broken one (remember that $q \simeq 0$ inside the vortex core).

From (3.23) we see that the energy increases with $R_{g}$ as $\left(\log L / R_{g}\right)^{-1}$ at $R_{g} \ll L$. Moreover, it is easy to estimate the surface energy of vortex if $R_{g} \gg L$. It goes as $v^{2} R_{g}$, thus infinite $R_{g}$ means infinite barrier.

Another possibility is that we have many bubbles of finite size which coalesce producing the unbroken vacuum. This means that we have many strings on the Higgs branch (they should be closed if monopoles are heavy). We discuss this possibility in subsection 4.3 . 
In the next subsection we study one more possibility for the string to develop instability. Imagine that the string develops large fluctuations actually covering the whole space. This may lead to the transition to the unbroken phase. To study this possibility we develop the string representation for the vortex.

\subsection{The string representation}

In this subsection we are going to find out if fluctuations of vortex string (deviation of vortex from a straight line) are controlled by any small parameter or not. To this end we develop string representation for the vortex. To do this we follow the logic of Refs. 21, 22, where the string representation for vortex in the London limit $\left(m_{H} \gg m_{\gamma}\right)$ has been developed. See also recent paper [23] where the string representation for the case $m_{H}=m_{\gamma}$ is suggested.

To work out the string representation for the vortex we integrate out photon multiplet and use $m_{\gamma}\left(R_{g}^{-1}\right.$ in $(3.24)$, more exactly) as a UV-cutoff for our string theory. This leads to the $\sigma$-model for the real matter field $\phi(x)$ (see (3.4)) with the flat metric and the constraint $\phi=0$ on the string worldsheet $z_{\mu}(\sigma)$ ․ We have for the partition function for the boson sector of the theory

$$
\begin{aligned}
Z & =\int D \phi(x) D z_{\mu}(\sigma) D \rho(\sigma) J(\phi) \times \\
& \times \exp \left\{-\int d^{4} x\left[\left(\partial_{\mu} \phi\right)^{2}+\int d^{2} \sigma \delta^{(4)}(x-z(\sigma)) i \rho(\sigma) \phi(x)\right]\right\} .
\end{aligned}
$$

Here we introduce field $\rho(\sigma)$ living on the string worldsheet as a Lagrange multiplier. The Jacobian $J(\phi)$ is defined by

$$
J(\phi) \int D z_{\mu}(\sigma) \prod_{\sigma} \delta(\phi(z))=1
$$

The Jacobian $J(\phi)$ is important for the quantization of the string theory. As it is argued in [24, 25] its role is to make the ANO string critical in four

\footnotetext{
${ }^{8}$ We also ignore $\phi_{2}, \phi_{3}$ and $\phi_{4}$ in (2.13) because they decouple from $\phi=\phi_{1}$ to the leading order.
} 
dimensions. We do not work out $J(\phi)$ here. It involves quantum corrections in the coupling constant of the $2 D \sigma$-model, which appears to be small as we show below.

Writing down $\phi=v+\delta \phi$ and integrating over $\delta \phi$ in (4.2) we get the $2 D$ theory on the string worldsheet

$$
\begin{aligned}
Z & =\int D z_{\mu} D \rho \exp -\left\{\int d^{2} \sigma i v \rho(\sigma)+\right. \\
& \left.+\int d^{2} \sigma d^{2} \sigma^{\prime} \frac{1}{4(2 \pi)^{2}} \frac{\rho(\sigma) \rho\left(\sigma^{\prime}\right)}{\left[z(\sigma)-z\left(\sigma^{\prime}\right)\right]^{2}}\right\} .
\end{aligned}
$$

The theory in (4.4) is a non-local one. It contains all powers of derivatives of $z_{\mu}(\sigma)$. To work out the leading term in derivatives let us fix the gauge

$$
z_{0}=\sigma_{1}, \quad z_{3}=\sigma_{2}
$$

and consider deviations from the flat string surface $z_{i}, i=1,2$ to be small. We have

$$
\left[z(\sigma)-z\left(\sigma^{\prime}\right)\right]^{2}=\left(\sigma-\sigma^{\prime}\right)^{2}+\left[\left(\sigma-\sigma^{\prime}\right)_{n} \partial_{n} z_{i}\right]^{2}+\cdots
$$

where $n=1,2$ runs over two coordinates in (4.5). Substituting (4.6) into (4.4) we get the integral

$$
\frac{1}{(2 \pi)^{2}} \int \frac{d^{2}\left(\sigma-\sigma^{\prime}\right)}{\left(\sigma-\sigma^{\prime}\right)^{2}}=\frac{1}{2 \pi} \ln m_{\gamma} L,
$$

where we use $m_{\gamma}$ as a UV-cutoff and characteristic size (length) of the string $L$ as a IR-cutoff. Using (4.7) and integrating over $\rho$ in (4.4) we get finally

$$
Z=\int D z_{\mu} \exp -\left\{\frac{2 \pi v^{2}}{\ln m_{\gamma} L}\left[T L+\int d^{2} \sigma \frac{1}{2}\left(\partial_{n} z^{i}\right)^{2}+\cdots\right]\right\} .
$$

The string tension in (4.8) coincides with the one in (1.3) which we have obtained in the last section solving classical equations of motion for the vortex (see (3.25)).

The action in the exponent in (4.8) is the expansion of Nambu-Goto action

$$
S_{\text {string }}=\tau \int d^{2} \sigma \sqrt{g},
$$


where $g_{m n}=\partial_{m} z_{\mu} \partial_{n} z_{\mu}$ is the induced metric and $\tau$ is given by (1.3). Corrections to (4.9) include higher derivative terms like rigidity term [2] and so on. However the expansion in derivatives hardly makes sense here because for $m_{H}=0$ the length of the string by itself plays the role of a IR-cutoff.

Now let us see whether our string is stable or it can develop large fluctuations. To this end let us estimate the renormalized string tension. We have

$$
\tau_{\text {ren }}=\tau-\frac{\text { const }}{R_{g}^{2}},
$$

where we use that the size of a core $R_{g}(3.24)$ plays the role of UV-cutoff for our string theory (4.9) ((4.4) more precisely).

Now let us show that the second term in (4.10) is small as compared to the first one. Its ratio to $\tau$ plays the role of the effective coupling constant in the $2 D \sigma$-model (4.9), (4.4)

$$
g_{2 D}^{2} \sim \frac{1}{R_{g}^{2} \tau} \sim \frac{g^{2}}{\ln m_{\gamma} L} .
$$

To estimate (4.11) let us recall our general strategy. We start with the microscopic non-Abelian Seiberg-Witten theory. Then we integrate over scales $\mu \gtrsim \Lambda$ and end up with effective $N=2$ QED (see (2.12)) near the root of the Higgs branch. The QED description is valid at scales $\mu$ in the region $m_{\gamma} \ll \mu \ll \Lambda$. The QED coupling constant $g^{2}$ is $1 / g^{2} \sim-\ln \mu / \Lambda_{Q E D}$, where $\Lambda_{Q E D}=m^{3} / \Lambda^{2}$ at $m \gtrsim \Lambda$. At the scale $\mu=m_{\gamma}$ the photon multiplet decouples. Thus the QED coupling in (4.11) is

$$
g^{2} \sim-\frac{1}{\ln \left(m_{\gamma} / \Lambda_{Q E D}\right)} .
$$

Below the photon mass at $\mu \ll m_{\gamma}$ we are left with the effective string theory (4.4) with the coupling (4.11). We see that $g_{2 D}^{2} \ll 1$. Thus we conclude that ANO string on the Higgs branch does not develop large fluctuations. It represents almost straight line connecting monopole with antimonopole. Its string tension approximately given by its classical value $\tau$ in (1.3).

It is worth noting that the rigidity term as well as higher corrections cause an additional renormalization of the string tension in (4.10) [26]. Still it is 
known [27, 26] that a $\sigma$-model without $\theta$-term has no IR-fixed point and its string tension remains finite.

This is usually considered as quite an unpleasant feature of the theory. Suppose we try to construct reasonable fundamental string theory starting from, say, random surfaces on a lattice using lattice spacing as a UV-cutoff. Then in order to get the string tension at some physical scale we need a fixed point for the $2 D \sigma$-model on the worldsheet. Moreover, the string tension should go to zero in lattice units in this fixed point [27].

In this paper we follow quite a different logic. We consider a solitonic ANO string rather than the fundamental one. The UV-cutoff for our string theory (4.4) is the photon mass $m_{\gamma}\left(R_{g}^{-1}\right.$ more precisely), which is already a physical parameter. The nonzero string tension given by (1.3) ensures confinement for monopoles in the conventional setup of this problem. Namely, for well separated $\left(L \gg R_{g}\right.$ ) heavy monopole-antimonopole pair its interaction potential (1.4) increased with $L$.

On the other hand, if we address the problem of calculating of the mass spectrum of hadrons on the Higgs branch then the string approach seems to be not applicable at all. 9 The reason for this is that the distance between monopole and antimonopole in the "meson" is $L_{0} \sim 1 / \sqrt{\tau}$ (at least for small spins). Thus $L_{0} \sim R_{g} \sim 1 / m_{\gamma}$. At that short distances string is not developed yet. We do not address problem of calculating of the hadron mass spectrum in this paper.

Let us note also, that because $1 / \sqrt{\tau}$ plays the role of the correlation length for the normals on the surface, nonzero $\tau$ means "crumpled" string surface [26, 28]. This is of course true only for open strings connecting light monopoles or for closed strings.

We considered only boson degrees of freedom of string in this subsection. In principle, one should add fermions as well. However, we do not expect any supersymmetry of our $2 D \sigma$-model (4.4) because our vortex is not BPS state. Fermions produce additional renormalization of the string tension in (4.10) which is of the same order $1 / R_{g}^{2}$ as the boson one. It is small as compared with classical $\tau$ and does not change our main conclusions.

\footnotetext{
${ }^{9}$ To address this problem with dynamical monopoles we have to go close to ArgyresDouglas point $m=\Lambda$ or consider closed strings to be interpreted as "glueballs".
} 


\subsection{Interactions of vortices}

Now we are going to study one another possibility for vortices to develop unstability and to destabilize the Higgs branch. We know from the condensed matter physics that vortices attract each other and coalesce producing the unbroken vacuum in the type one superconductor. To clarify this issue we now calculate interactions of vortices on the Higgs branch.

To do this we use the string representation (4.4). Suppose we have two parallel vortices of length $L$ at the separation $R, R \ll L$. Then to the leading order in $g_{2 D}^{2}$ the contribution of these two vortices to the partition function is

$$
\begin{aligned}
Z_{1,2} & =\int D z_{1} D z_{2} D \rho_{1} D \rho_{2} \exp -\left\{\int d^{2} \sigma_{1}\left[i v \rho_{1}+\frac{\rho_{1}^{2}}{4} \frac{1}{2 \pi} \ln m_{\gamma} L\right]\right. \\
& +\int d^{2} \sigma_{2}\left[i v \rho_{2}+\frac{\rho_{2}^{2}}{4} \frac{1}{2 \pi} \ln m_{\gamma} L\right]+ \\
& \left.+\int d^{2} \sigma_{1} d^{2} \sigma_{2} \frac{1}{4} \rho_{1} \rho_{2} \frac{1}{(2 \pi)^{2}} \frac{1}{\left[z_{1}\left(\sigma_{1}\right)-z_{2}\left(\sigma_{2}\right)\right]^{2}}\right\},
\end{aligned}
$$

where $z_{1 \mu}\left(\sigma_{1}\right), \rho_{1}\left(\sigma_{1}\right)$ and $z_{2 \mu}\left(\sigma_{2}\right), \rho_{2}\left(\sigma_{2}\right)$ describes the first and the second vortex respectively. We neglect fluctuations of vortices in (4.13) and fix the gauge (4.5) for each vortex. Two first terms in the exponent in (4.13) correspond to each individual vortex while the third one describes their interactions. Performing the integral over $\left(\sigma_{1}-\sigma_{2}\right)$ in (4.13) and integrating out $\rho_{1}$ and $\rho_{2}$ we get for the vortex interaction potential

$$
U_{1,2}(R)=-2 \pi^{2} v^{2} T L \frac{\ln L / R}{\left(\ln m_{\gamma} L\right)^{2}} .
$$

The potential (4.14) shows the logarithmic attraction. It is clear that if we consider the case $R \gg L$ then the problem becomes three dimensional and $U_{1,2} \sim-1 / R$. Note also, that the vortex-antivortex interaction is the same as the vortex-vortex one in the limit $m_{H}=0$.

Thus it is clear that vortices interact via attractive Coulomb forces $(U \sim$ $\ln R$ if $R \ll L$ and $U \sim-1 / R$ if $R \gg L$ ). However, we know that the Coulomb gas with atraction is unstable. This means that if vortices condense then they attract each other and coalesce destabilizing the Higgs branch. 
This is exactly what happens in the condensed matter superconductor when the external magnetic field exceeds its critical value.

To be more specific we can describe the interaction of the string with the external Higgs field using eq.(4.2). It has the form

$$
\int d^{4} x i \phi(x) j(x),
$$

where $j(x)=\int d^{2} \sigma \delta^{(4)}(x-z(\sigma)) \rho(\sigma)$ is the "string current". The condensation of strings means that $j$ develops nonzero VEV. From (4.15) we see that this would destabilize the Higgs branch.

Thus it is clear that the problem is whether vortices condense or not. Now we are going to argue that vortices do not condense and the Higgs branch remains stable. First note, that open strings could condense only if monopoles condense. However we know that monopoles do not condense even at the Argyres-Douglas point (where they become massless) because we have only one flavor of monopoles and Eqs. (2.6) and (2.7) have no solutions for monopoles. Still this argument does not rule out the possibility of condensation of closed strings.

The more general argument is based on $N=2$ supersymmetry. From (4.15) we expect that if vortices condense they would generate a superpotential

$$
\int d^{2} \theta m_{0} \widetilde{Q} Q,
$$

where $m_{0}$ is proportional to $\langle j\rangle$. Note that the mass term is the only superpotential for matter fields which is allowed by the $N=2$ supersymmetry ( if gauge fields are absent, see, for example, review [29). However, (4.16) does not destabilize the Higgs branch. It just produce a shift in the bare mass parameter $m$.

This argument has even more general nature. If vortices destabilize the Higgs branch we expect them to generate some superpotential on it. As soon as no non-trivial superpotential is allowed without breaking $N=2$ supersymmetry we conclude that the Higgs branch is stable. This means that vortices do survive in the quantum theory giving rise to the confining potential (1.4) between heavy well separated monopoles on the Higgs branch. 


\section{Conclusions}

In this paper we studied vortices on the Higgs branch of the Seiberg-Witten model. We showed that vortices of finite length exist classically and produce the confining potential (1.4) for heavy monopoles. This confining potential is not linear in $L$ and we can use Wilson loop as an order parameter to distinguish this new confining phase specific for Higgs branches in supersymmetric theories.

Then we studied the stability of vortices in the quantum theory. First, we showed that vortices do not grow in their transverse size $R_{g}$. Second, we studied vortices in the string representation and showed that ANO strings do not develop large fluctuations and their string tension remains nonzero in the quantum theory.

Next we ruled out the possibility for vortices to condense and form the Coulomb gas with attraction which would destabilize the Higgs branch. Our conclusion is that vortices survive in the quantum theory producing a new type of confining phase for monopoles with the potential (1.4).

We have not addressed the problem of calculating of the hadron mass spectrum on the Higgs branch. As we have shown the string approach is not acceptable for this purpose. Still it is quite plausible to suggest on the general grounds that the lightest monopole-antimonopole "meson" becomes massless at the Argyres-Douglas point $m=\Lambda$. To see the reason for this let us recall that the root of a Higgs branch at $m=\Lambda$ corresponds to conformal field theory with non-trivial anomalous dimensions [18. If all "hadrons" built of monopoles are massive at this point we could integrate them out together with photon multiplet at scales $\mu^{2} \ll\langle\bar{q} q\rangle$. Then we would get a $\sigma$-model for charges $q_{\alpha}$ with the flat metric (the Higgs branch admits only unique hyperKahler metric). Then nothing prevents us from taking the limit $\langle\bar{q} q\rangle \rightarrow 0$, in which we would get trivial fixed point. This is correct for all values of mass parameter except Argyres-Douglas points $m= \pm \Lambda$, where we should recover non-trivial conformal field theory.

The possible resolution of this puzzle is that there is a massless monopole (dyon) "meson" at $m=\Lambda(-\Lambda)$ (see also [14). Still the calculation of the "hadron" mass spectrum on the Higgs branch remains to be an open problem. 


\section{Acknowledgments}

The author is grateful to A. Gorsky, A. Marshakov, V. Rubakov, and A. Schwimmer for useful discussions. In particular, the author would like to thank A. Vainshtein for numerous and very illuminating discussions. Also the author is grateful to the Theoretical Physics group at the University of Minnesota where a part of this work has been done for hospitality. This work is supported by Russian Foundation for Basic Research under grand No. 9902-16576. 


\section{References}

[1] C. Montonen and D. Olive, Phys.Lett.B 72 (1977) 117;

P. Goddard, J. Nuyts and D.Olive, Nucl.Phys.B 125 (1977) 1.

[2] N. Seiberg and E. Witten, hep-th/9407087; Nucl.Phys.B 426 (1994) 19.

[3] N. Seiberg and E. Witten, hep-th/9408099; Nucl.Phys.B 431 (1994) 484.

[4] S. Mandelstam, Phys.Rep. 23C (1976) 145;

G. t'Hooft, in Proceed.of the Europ.Phys.Soc. 1975, ed.by A.Zichichi (Editrice Compositori, Bologna, 1976), p.1225.

[5] A.A. Abrikosov, Sov.Phys.JETP, 32 (1957) 1442;

H.B. Nielsen and P. Olesen, Nucl.Phys. B61 (1973) 45.

[6] W. Fuertes and J. Guilarte, hep-th/9807218; Phys.Lett.B 437 (1998) 82 .

[7] E.B. Bogomolny, Sov.J.Nucl.Phys. 24 (1976) 449.

[8] Z. Hlousek and D. Spector, Nucl.Phys.B 370 (1992) 143;

J. Edelstein, C.Nunez, and F. Schaposnik, hep-th/9311055; Phys.Lett. 329B (1994) 39.

[9] A. Hanany, M. Strassler, and A. Zaffaroni, hep-th/9707244; Nucl.Phys.B 513 (1998) 87.

[10] E.B. Bogomolny and A.I. Vainshtein, Sov.J.Yad.Fiz. 23 (1976) 1111.

[11] A. Penin, V. Rubakov, P. Tinyakov and S. Troitsky, hep-th/9609257; Phys.Lett B 389 (1996) 13.

[12] D.Finnell and P. Poliot, hep-th/9503115, Nucl.Phys.B 453 (1995) 225.

[13] P. Argyres, M. Plesser and N. Seiberg, hep-th/9603042; Nucl.Phys.B 471 (1996) 159.

[14] A. Yung, hep-th/9705181; Nucl.Phys.B 512 (1998) 79. 
[15] O. Aharony, A. Hanany, K. Intriligator, N. Seiberg, and M. Strassler, hep-th/9703110; Nucl.Phys.B 499 (1997) 67.

[16] B.R. Greene, D.R. Morrison and C. Vafa, hep-th/9608039; Nucl.Phys.B 481 (1996) 513.

[17] P.C. Argyres and M.R. Douglas, hep-th/9505062; Nucl.Phys.B 448 (1995) 93.

[18] P.C. Argyres, M.R. Plesser, N. Seiberg, and E. Witten, hep-th/9511154; Nucl.Phys.B 461 (1996) 71.

[19] A. Bilal and F. Ferrari, hep-th/9706145; Nucl.Phys.B 516 (1998) 175.

[20] A. Hanany and I. Klebanov, hep-th/9606136; Nucl.Phys.B 482 (1996) 105.

[21] P. Orland, hep-th/9404140; Nucl.Phys.B 428 (1994) 221.

[22] M. Sato and S. Yahikozawa, hep-th/9406208; Nucl.Phys.B 436 (1995) 100.

[23] M. Baker and R. Steinke, "An effective string theory of AbrikosovNielsen-Olesen vortices", hep-th/9905375.

[24] J. Polchinski and A. Strominger, Phys.Rev.Lett. 67 (1991) 1681.

[25] E. Akhmedov, M. Chernodub, M. Polikarpov, and M. Zubkov, Phys.Rev.D 53 (1996) 2087.

[26] A. Polyakov, Nucl.Phys.B 268 (1986) 406.

[27] B. Durhuus, J. Frohlich and T. Jonsson, Nucl.Phys.B 240 (1984) 453;

J. Ambjorn, B. Durhuus and J. Frohlich, Nucl.Phys.B 270 (1986) 433;

P. Orland, Nucl.Phys.B 275 (1986) 457.

[28] G. David, Phys.Rep. 184 (1989) 221.

[29] M. Sohnius, Phys.Rep. 128 (1985) 39. 